\title{
Stretching and Tunability of Graphene-Based Passive Terahertz Components
}

\author{
Konstantin G. Batrakov,* Nadezhda I. Volynets, Alesia G. Paddubskaya, \\ Polina P. Kuzhir, Maria Stella Prete, Olivia Pulci, Evgeni Ivanov, Rumiana Kotsilkova, \\ Tommi Kaplas, and Yuri Svirko
}

The dependence of transmission/absorption of terahertz ( $\mathrm{THz}$ ) radiation on strain in graphene is investigated experimentally and with the aid of $a b$ initio calculations. By applying strain to chosen graphene layer(s), the effective sheet conductance can be fine-tuned to necessary value to design tunable passive $\mathrm{THz}$ components (such as shields, filters, polarizers, etc.) utilizing the high absorption ability of graphene. The positive influence of nonperfectness of chemically vapor deposited (CVD) graphene for strong tunability versus mechanical deformations is also discussed.

\section{Introduction}

Our daily life heavily relies on sunlight. However, the spectrum of the electromagnetic (EM) waves we use is much broader. It spans from $50 / 60 \mathrm{~Hz}$, the frequency of the power supply in our households, to X-rays, which we use for routine medical screening. This vast range includes microwave

Dr. K. G. Batrakov, N. I. Volynets, A. G. Paddubskaya, Dr. P. P. Kuzhir Research Institute for Nuclear Problems

Belarusian State University

Minsk 220006, Belarus

E-mail: kgbatrakov@gmail.com

Dr. K. G. Batrakov, A. G. Paddubskaya, Dr. P. P. Kuzhir

Tomsk State University

Tomsk 634050, Russia

Dr. M. S. Prete, Prof. O. Pulci

Department of Physics, and INFN

University of Rome Tor Vergata

Rome, Italy

Prof. E. Ivanov, Prof. R. Kotsilkova

Institute of Mechanics

Bulgarian Academy of Science

Sofia, Bulgaria

Dr. T. Kaplas

Department of Optoelectronics

Center for Physical Sciences and Technology

Saulètekio av. 3, LT-10257 Vilnius, Lithuania

Dr. T. Kaplas, Prof. Y. Svirko

Department of Physics and Mathematics

University of Eastern Finland

Joensuu FI-80101, Finland

The ORCID identification number(s) for the author(s) of this article can be found under https://doi.org/10.1002/pssb.201800683.

DOI: 10.1002/pssb.201800683 (communication), infrared (night vision) and optical waves. Nevertheless, there exists a narrow band between microwave and infrared that has been not in use until recently. This is the so-called Terahertz $(\mathrm{THz})$ gap, which is still hardly reachable by either electronic or optical devices. This frequency range corresponds to the temperature of biological processes and covers a substantial fraction of the luminosity from the Big Bang. The emergence of novel $\mathrm{THz}$ sources, such as quantum cascade lasers, opened the path to the exploitation of THz waves. ${ }^{[1]}$ The growing interest over the past 10 years in THz science is due to its many important applications in physics, astronomy, chemistry, biology, and medicine, including $\mathrm{THz}$ imaging, microscopy, non-destructive testing, tomography, medical diagnosis, health monitoring, environmental control, chemical and biological identification, and in future communication networks. These applications require efficient, durable, robust, and tunable components capable of generating, manipulating, and detecting of $\mathrm{THz}$ radiation. ${ }^{[2]}$ According to the just published The 2017 terahertz science and technology roadmap ${ }^{[3]}$ among the most important challenges are passive quasi-optical components, i.e., filters, polarizers, collimators, and modulators.

Conventional optical components can be used in the $\mathrm{THz}$ range. However, the performances of $\mathrm{THz}$ quasi-optics have not reached the same levels as at optical, much shorter, wavelengths. Most popular material solution for passive components at $\mathrm{THz}$ is silicon. However, since the undoped $\mathrm{Si}$ possesses a high refractive index, 3.41 at $\mathrm{THz}, \mathrm{Si} /$ air interface reflects $32 \%$ of the incident radiation thus decreasing significantly the efficiency of filters, polarizers, etc. What is more, the main drawback of existing $\mathrm{THz}$ components is their restricted tunability, which is the must for future $\mathrm{THz}$ devices.

Graphene, being light, flexible, and shatterproof, is very attractive to be used in electronic components in post-silicon age. Electromagnetic properties of graphene and its ability to absorb radiation strongly depend on the frequency range, i.e., on which transitions (inter- or intra-band) ${ }^{[4]}$ dominate the graphene ac conductivity. In the visual and IR range, graphene monolayer absorbs $2.3 \%$ of incident light mainly due to inter-band transitions, while in the $\mathrm{THz}-\mathrm{subTHz}$ range, the intra-band processes dominate giving rise to absorptance of as high as $20+\%$ per graphene sheet, depending on the doping level. 
Recently, we have demonstrated ${ }^{[5]}$ that free standing multilayered structures consisting of alternating graphene monolayers and submicron-thin polymer (PMMA) slabs are capable to absorb up to $50 \%$ of the radiation, depending on the number of graphene sheets, thus providing an efficient microwave shielding. According to admittance-matching conditions, in case of using a proper dielectric substrate being $1 / 4$ of wavelength thick, ${ }^{[6]}$ or metamaterials-like type ${ }^{[7]}$ it is possible to reach almost perfect absorption. The concept of EM components relying on graphene high absorption ability in microwave- $\mathrm{THz}$ frequency range has been proposed in our recent work: high performance of graphene in shields, filters, polarizers, operating at the $\mathrm{GHz}$ and $\mathrm{THz}$ frequencies has also been recently demonstrated. ${ }^{[8]}$

In order to achieve tunability, we may utilize one of the many outstanding properties of graphene ${ }^{[9-12]}$ for example its ability to tune the Fermi level with external doping or biasing. Graphene transmission/absorption coefficients depend, in particular, on chemical potential $\mu .^{[4,6]}$ For chemical vapor deposition (CVD)produced graphene, typical values of the chemical potential are $|\mu| \sim 0.1 \div 0.2 \mathrm{eV}$. Density of carriers and, therefore, graphene sheet conductance depend also on temperature. Hence, transmittance/absorbance can be tuned by temperature variation. However, a rather high variation of temperature is needed for noticeable carrier density altering in CVD graphene.

One more method to tune electronic and optical properties of graphene is to apply mechanical strain or bending. ${ }^{[13,14]}$ In fact, among the many outstanding characteristics, graphene posses also unique mechanical properties with elastic reversible resistance to tensile stress. ${ }^{[15]}$ Landau levels and optical Hall effect have been extensively studied in strained graphene. ${ }^{[16]}$ Effect of strain has been widely investigated with the aim to open a gap (through high shear and uniaxial strain), to produce very strong pseudomagnetic fields (non-uniform strain) and to break the electron-hole symmetry giving rise to a non-isotropic Dirac cone and hence a non-universal IR absorbance for uniaxial strain. $^{[17]}$

Here, we study the possibility to tune the transmittance ability of graphene/PMMA sandwich structure (i.e., to tune passive $\mathrm{THz}$ components relying on the absorbance ability of graphene) with isotropic stretching or strain. Mechanical deformations could be an interesting alternative to other widely applied ways of using external forces to change the chemical potential, mobility and relaxation time of graphene, e.g., electrostatic doping, ${ }^{[18-23]}$ allowing to avoid widely discussed problems of high and nonreproducible metal-graphene contact resistance. ${ }^{[24-31]}$

\section{Theory}

As shown in ref. [5], maximal absorptance in graphene corresponds to some optimal value of the effective sheet conductance. Such an optimal value can be reached by combining several graphene layers in sandwich structure. ${ }^{[5,6]}$ Fine-tuning to the exact value of optimal conductance can be fulfilled by applying strain to one of the layers. Sheet conductance depends on the carrier density $n$, which is connected to the chemical potential $\mu$ and relaxation time $\tau$ due to carrier scattering processes. The dominant contribution to carrier scattering in supported CVD graphene (on the substrate, e.g., on the PMMA layer) is due to charge impurities and defects. ${ }^{[32,33]}$ In contrast, in high quality suspended graphene, mobilities exceeding $200000 \mathrm{~cm}^{2} \mathrm{~V}^{-1}$ are obtained, with main contribution to carrier scattering arising from electron-phonon interaction.

The dependence of chemical potential on strain can be extracted from the considerations below. Carriers density changes with $\mu$ following the equation

$\int \frac{k d k}{(2 \pi)^{2}} \frac{g_{\mathrm{s}} g_{\mathrm{v}}}{\exp \{(\varepsilon-\mu) / T\}+1}=\frac{n_{0}}{(1+\sigma)^{2}}$

Here $g_{s}, g_{v}$ are spin and valley degeneracy, for graphene these parameters equal to two, $n_{0}$ is carrier density of unstrained graphene, $\sigma$ is linear strain, $k$ and $\varepsilon$ are electron momentum and energy correspondingly.

In reality, when $|\mu| \gg T$ (typical situation for CVD graphene and room temperature), and $|\mu| \ll t$, where $t$ is graphene hopping parameter, Eq. (1) could be simplified

$\frac{g_{\mathrm{s}} g_{\mathrm{v}} \mu^{2}}{2(2 \pi)^{2} v_{\mathrm{F}}^{2}}=\frac{n_{0}}{(1+\sigma)^{2}}=\frac{g_{\mathrm{s}} g_{\mathrm{v}} \mu_{0}^{2}}{2(2 \pi)^{2} v_{\mathrm{F} 0}^{2}(1+\sigma)^{2}}$

or

$\frac{\mu}{\mu_{0}}=\frac{v_{\mathrm{F}}}{v_{\mathrm{F} 0}(1+\sigma)}$

When deriving (3) the step Dirac-Fermi distribution $(|\mu| \gg T)$ and Dirac dispersion law for $\pi$ electrons in graphene $(|\mu| \ll t)$ were used for electrons near the Dirac point. Equation (1) or (3) set the value of the chemical potential, which is necessary for transmission/absorption calculations. $v_{\mathrm{F}_{0}}$ and $v_{\mathrm{F}}$ are Fermi velocities for unstrained and strained graphene, respectively.

Our calculations for the dependence of the Fermi velocity on strain are based on density functional theory (DFT) ${ }^{[34,35]}$ using the Quantum ESPRESSO code. ${ }^{[36]}$ The exchange and correlation functional was selected to be the Perdew-Burke-Ernzerhof (PBE).$^{[37]}$ The calculated Fermi velocities are reported in Table 1, together with the lattice constants and work functions. A clear trend is found: by increasing the strain, the Fermi velocities decrease, and the work function increases. Another parameter which influence conductance is relaxation time. Expressions for it, considering that charge impurities give main contribution,

Table 1. Calculated lattice constant $\left(a_{\text {lat }}\right)$, Fermi velocity, and work function (WF) of graphene for different strain isotropic on the $x y$ plane.

\begin{tabular}{lccc}
\hline Strain & $a_{\text {lat }}(\mathrm{A})$ & $\nu_{\mathrm{F}}\left(10^{6} \mathrm{~m} \mathrm{~s}^{-1}\right)$ & $\mathrm{WF}(\mathrm{eV})$ \\
\hline 0.0 & 2.460 & 0.844 & 4.22 \\
$2.2 \%$ & 2.513 & 0.819 & 4.40 \\
$2.4 \%$ & 2.518 & 0.813 & 4.42 \\
$2.6 \%$ & 2.523 & 0.811 & 4.43 \\
$3.5 \%$ & 2.546 & 0.805 & 4.51 \\
$4.8 \%$ & 2.576 & 0.785 & 4.61 \\
$5.5 \%$ & 2.595 & 0.783 & 4.65 \\
\hline
\end{tabular}


was derived, for example, in ref. [33]

$\frac{1}{\tau}=\frac{n_{\mathrm{i}}}{2 \pi \hbar}\left(\frac{2 \pi e^{2}}{\kappa}\right)^{2} \frac{2 I_{0}}{\mu}$

Here $n_{\mathrm{i}}$ is impurity concentration, $k$ is substrate (or interface) dielectric constant, renormalized by graphene polarization ${ }^{[32]}$

$I_{0}=\int_{0}^{1} d x \frac{x^{2} \sqrt{1-x^{2}}}{\left(x+2 r_{\mathrm{s}}\right)^{2}}$

$r_{\mathrm{S}}=e^{2} / \kappa \hbar v_{\mathrm{F}}$ is graphene fine structure constant. Relaxation time increases with chemical potential (carrier concentration) increase. It is caused by screening effect. Electron cloud screens charged impurities and diminishes long-range scattering. The chemical potential and carrier concentration drop during tensile strain (3). This factor decreases relaxation time. Strain also affects the fine structure constant which increases (because $v_{\mathrm{F}}$ decreases). This factor changes $\tau$ in different direction during tensile strain, because decrease of Fermi velocity decreases screening distance. Resulting relaxation time dependence on strain without considering change of impurity concentration can be derived using expressions (3)-(5). Figure 1 demonstrates dependence of $\tau(\sigma) / \tau_{0}$ on $\sigma$. The dependence for hopping parameter $t=t_{0} \exp (-3.37 \sigma)^{[38]}$ was used. This formula slightly overestimates the effect of strain on hopping parameter.

Table 1 presents ab initio calculation for some values of strain applied isotropically to graphene including 2.2, 2.4, 2.6, 3.5, 4.8, and $5.5 \%$ in experiments. The strain produces a change in the Fermi velocity and in the work function (WF). In particular, with increasing strain (and hence, increasing C-C distance) the Fermi velocity decreases (see Table 1). According to our DFT calculations, even in presence of strain, the most stable geometry is still planar (no buckling).

The Dirac cone survives also in presence of the imposed strain (see Figure 2). As a consequence, the infrared absorbance (interband transitions), which for linear bands in 2D system tends to the universal constant $\pi a^{[39-41]}$ ( $\alpha$ is the Sommerfeld fine structure constant), does not change with the application of

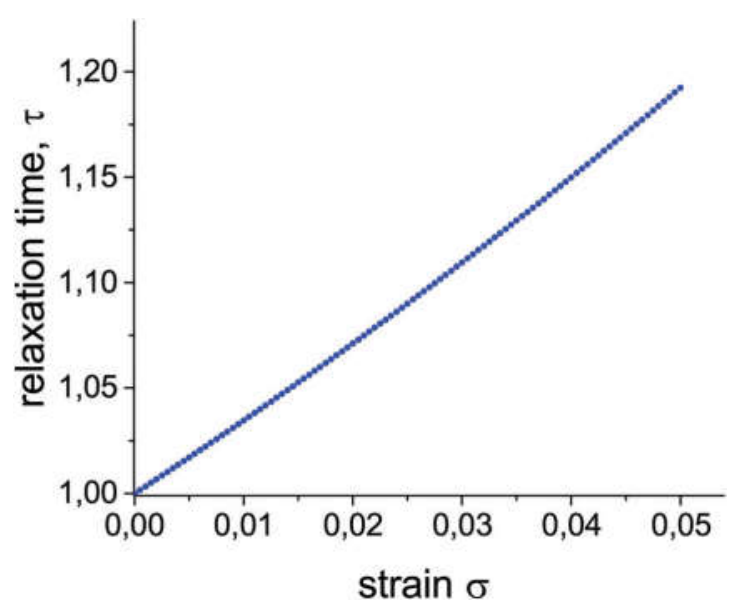

Figure 1. Dependence of relaxation time on strain.

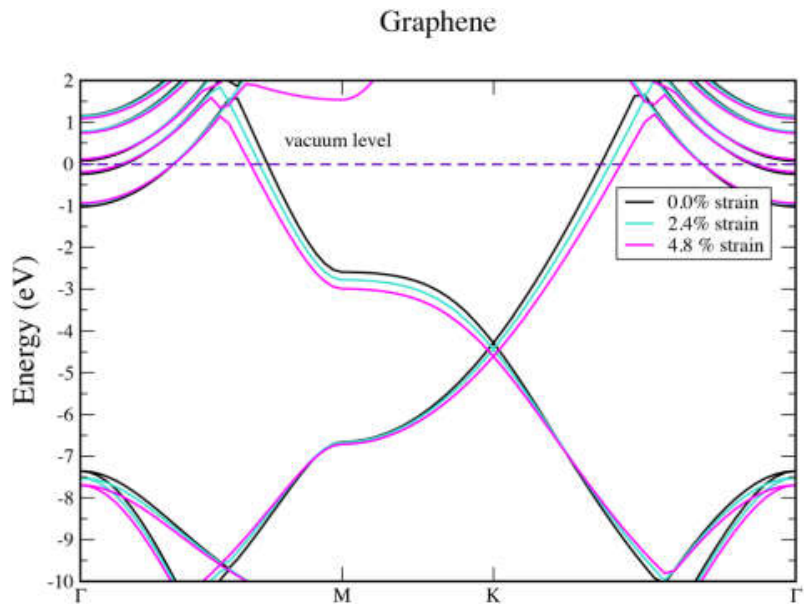

Figure 2. Electronic band structure of graphene (DFT calculation). The bands for unstrained graphene and for strained graphene (2.4 and $4.8 \%$ ) have been aligned to the same vacuum level. The Fermi energy (and hence the Work Function) change with changing the applied strain.

strain. Interestingly, there are instead large changes in the absorbance and in the 2D-optical conductivity for higher photon energies (see Figure 2 and Figure 3).

\section{Experimental Section}

Graphene was synthetized by conventional CVD method on a copper foil ( $25 \mu \mathrm{m}, 99.8 \%$ pure) by using a custom-made CVD reactor. The reactor chamber was pumped to vacuum for $1 \mathrm{~h}$ to cleanse air and moisture from the chamber. Thereafter, the chamber with the foil was heated to $1000^{\circ} \mathrm{C}$ in $\mathrm{H}_{2}$ flow $(5 \mathrm{sccm}$, $0.1 \mathrm{mbar}$ ) and the $\mathrm{Cu}$ foil was annealed $30 \mathrm{~min}$ at this temperature. After the $\mathrm{Cu}$ foil annealing, graphene was synthetized on the foil by injecting $\mathrm{CH}_{4}(5 \mathrm{sccm})$ together with $\mathrm{H}_{2}(5 \mathrm{sccm})$ into the chamber. The graphitization part lasted $15 \mathrm{~min}$ and afterwards,

Graphene

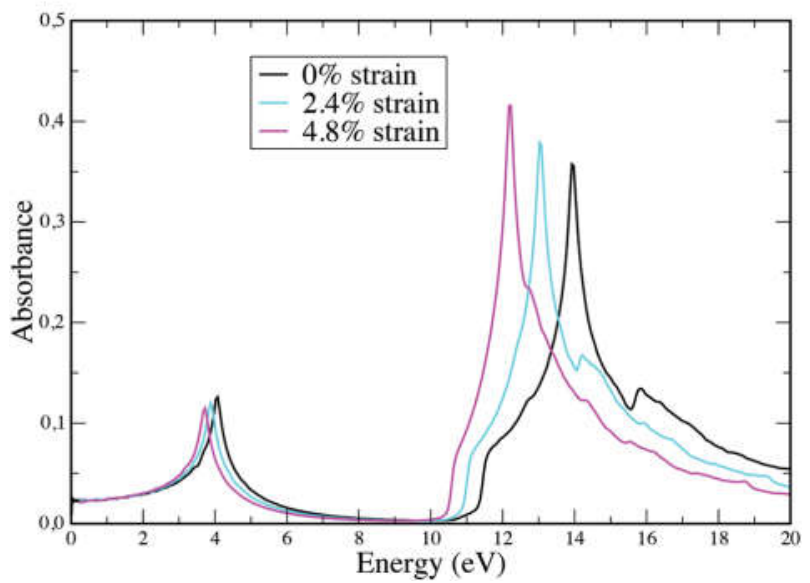

Figure 3. Two-dimensional absorbance for different values of applied strain. Just interband transitions are included. 
the chamber was filled with $\mathrm{H}_{2}(10 \mathrm{mbar})$ and the chamber was cooled down to room temperature (overnight).

The $\mathrm{Cu}$ foil with graphene was spin coated with a one micron thick poly(methyl methacrylate) (PMMA) layer, which was cured in $60 \mathrm{C}$ on a hot-plate. The backside of the $\mathrm{Cu}$ foil was treated with oxygen plasma $(20 \mathrm{~s} / 100 \mathrm{~W} / 20 \mathrm{sccm})$ to remove graphene layer which was grown on another side of the foil. After removing backside graphene, the $\mathrm{Cu}$ foil was etched by $\mathrm{FeCl}_{3}$ solution (overnight). The PMMA-graphene layer was rinsed twice in water before it was attached on a metal plate with a hole (diameter of $10 \mathrm{~mm}$ ). An adhesion between the PMMA-graphene sandwich and the metal plate was promoted by a double-sided tape around the hole, which allowed us to apply mechanical stress to the sandwich structure.

It is demonstrated from stress-induced Raman bands shifts that stress can be transferred from a polymer matrix to a graphene monolayer in a system polymer/graphene. ${ }^{[42]}$ Here we deal with the same situation.

Terahertz response on isotropic strain was studied. For stretching, contact loading small punch test experiments were done (see for details ref. [43]). Briefly, the Universal Mechanical Testing device UMT-2M (Bruker) with small punch setups (equipped with a very sensitive sensor for load $0.1 \mathrm{mN}$ working in both load control and displacement control modes) was used for macromechanical characterization of the freestanding graphene/PMMA sandwich. The punch diameter was of $6 \mathrm{~mm}$ with a spheroidal end shape. Table 2 presents the loadtest details. The graphene/PMMA samples were stretched by 2.2 and $2.6 \%$, respectively.

At $\mathrm{THz}$ frequency range, the EM response of unstrained and strained samples has been measured using a commercial $\mathrm{THz}$ time-domain spectrometer "T-Spec" by EKSPLA. In a setup photoconductor antenna (low temperature grow GaBiAs), illuminated by ultrashort laser pulses with $1050 \mathrm{~nm}$ wavelength, 50-150 fc pulse duration and more than $40 \mathrm{~mW}$ output power, was used in order to generate $\mathrm{THz}$ radiation up to $2 \mathrm{THz}$. The $\mathrm{THz}$ radiation was focused onto the sample by parabolic mirrors. THz pulses are detected in a similar photoconductive antenna. In order to get better signal to noise ratio (more than 106:1 at $0.4 \mathrm{THz}$ with spectral resolution $5 \mathrm{GHz}$ ) up to 1024 scanned curves were averaged for each measurement. This allows us to obtain the experimental error less than $2 \%$. Fourier transformation was used to convert the time-domain signal into the frequency domain.

Table 2. The load-test details.

\begin{tabular}{|c|c|}
\hline Sample & Mechanical stretching test details \\
\hline $\begin{array}{l}\text { Graphene/PMMA, } \\
2.2 \% \text { strain }\end{array}$ & $\begin{array}{l}\text { The Mullins-type } 3 \text {-step load-upload stretching test runs at } \\
1 \mathrm{mN} \text { min }^{-1} \text { load rate at } 3 \text { steps of increasing load, up to } \\
\text { maximum of } 6 \mathrm{mN} \text {, at } 2 \text { min running time for each step. } \\
\text { After every load step, a fast upload for } 1 \mathrm{~s} \text {, followed by a } \\
\text { hold at } 0.3 \mathrm{vmN} \text { for } 2 \mathrm{~min} \text { is applied }\end{array}$ \\
\hline $\begin{array}{l}\text { Graphene/PMMA, } \\
2.6 \% \text { strain }\end{array}$ & $\begin{array}{l}\text { The } 3 \text {-step load-hold stretching test runs at } 1 \mathrm{mN} \mathrm{min}^{-1} \\
\text { load rate up to maximum load of } 6 \mathrm{mN} \text {, as divided in } \\
3 \text { steps of equal load each and } 6 \mathrm{~min} \text { running time. After } \\
\text { every load step, a hold at the respective peak load is } \\
\text { applied for } 2 \mathrm{~min}\end{array}$ \\
\hline
\end{tabular}

\section{Results and Discussion}

Figure 4a demonstrates experimental transmission data and calculated ones for unstrained samples and graphene/PMMA stretched by 2.2 and $2.6 \%$. One can see that small strain (stretching) up to $2.6 \%$ leads to significant increase of transmittance ability of graphene/PMMA sandwich in $\mathrm{THz}$ range (from 62 to $72 \%$ ).

Comparing experimental data for as-prepared sample with theoretical calculations using expression (3)-(5), one may conclude that concentration of charged impurities in unstrained graphene is about $10^{11} / Z^{2} \mathrm{~cm}^{-2}$, where $Z e$ is the charge of impurity. The same procedure for strained graphene/PMMA shows that additional micro-defects and impurities appear during the strain as impurities concentration increases by $25 \%$ at strain $2.2 \%$ and by $40 \%$ at $2.6 \%$. It worth noting that within a range of experimental error there is a good agreement between
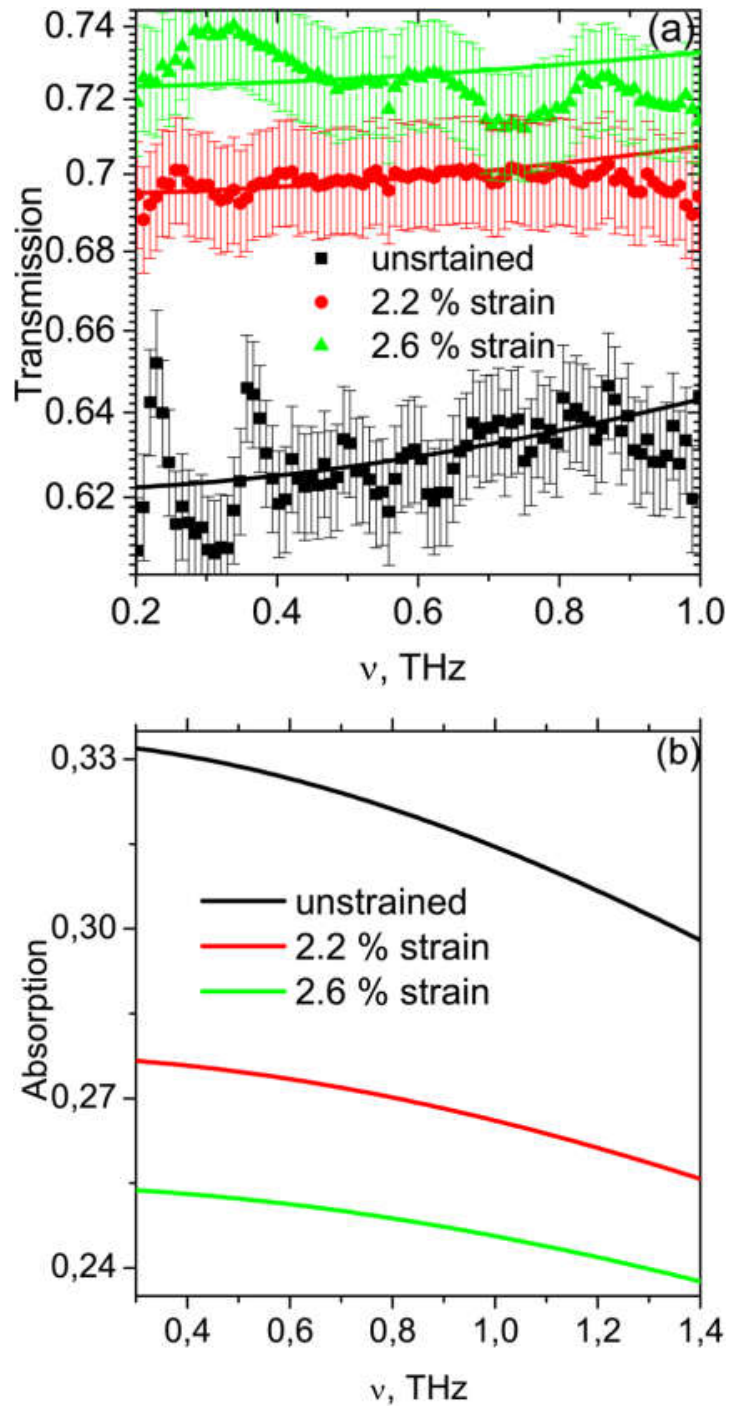

Figure 4. Frequency dependence of the transmittance (a) and absorbance (b); measured (symbols) and calculated values for the graphene of different strain. 
transmission spectra measured experimentally and calculated using the theoretical model.

\section{Conclusions}

Our conclusions are summarized by Figure 4b, which presents calculated absorptance of graphene/PMMA sandwich versus applied strains. The absorbance changes with the applied external forces, from $33 \%$ to $23-25 \%$. That is starting from observation of basic principles of mechanically induced tunability concept, we show possibility to tune $\mathrm{THz}$ response of passive $\mathrm{THz}$ components made of CVD graphene by small mechancal deformations. The importance of CVD graphene imperfectness, i.e., the positive influence of different graphene micro-defects and impurities and their dynamics on the high level of $\mathrm{THz}$ graphene tunability at small strain is also addressed, opening the route to cost-effective and efficient processing of tunable passive components necessary for future and emerging $\mathrm{THz}$ technologies.

\section{Acknowledgments}

This publication was supported by H2020-CA644076 RISE project CoExAN, H2020-MSCA-RISE-2016-734164 Graphene 3D, DiSeTCom H2020 RISE Project number: 823728 and the Union State TechnologySG programme. KGB, AGP, and PPK are thankful to Tomsk State University competitive programme. P.K. is thankful to grant of the president of the republic of Belarus in science, education, health, culture in 2019.

\section{Conflict of Interest}

The authors declare no conflict of interest.

\section{Keywords}

absorption, Fermi velocity, graphene, graphene sandwich, strain, terahertz

Received: November 15, 2018 Revised: March 6, 2019 Published online:

[1] B. S. Williams, Nat. Photon. 2007, 1, 517.

[2] T. Nagatsuma, G. Ducournau, C. C. Renaud, Nat. Photon. 2016, 10, 371.

[3] S. S. Dhillon, M. S. Vitiello, E. H. Linfield, A. G. Davies, M. C. Hoffmann, J. Booske, C. Paoloni, M. Gensch, P. Weightman, G. P. Williams, E. Castro-Camus, D. R. S. Cumming, F. Simoens, I. Escorcia-Carranza, J. Grant, S. Lucyszyn, M. Kuwata-Gonokami, K. Konishi, M. Koch, C. A. Schmuttenmaer, T. L. Cocker, R. Huber, A. G. Markelz, Z. D. Taylor, V. P. Wallace, J. A. Zeitler, J. Sibik, T. M. Korter, B. Ellison, S. Rea, P. Goldsmith, K. B. Cooper, R. Appleby, D. Pardo, P. G. Huggard, V. Krozer, H. Shams, M. Fice, C. Renaud, A. Seeds, A. Sttohr, M. Naftaly, N. Ridler, R. Clarke, J. E. Cunningham, M. B. Johnston, J. Phys. D: Appl. Phys. 2017, 50, 043001.

[4] Carbon Nanotubes and Graphene for Photonic Application (Eds: J. H. Choi, S. Yamashita, Y. Y. Saito), Woodhead Publishing, Cambridge, UK 2013.

[5] K. Batrakov, P. Kuzhir, S. Maksimenko, A. Paddubskaya, S. Voronovich, P. Lambin, T. Kaplas, Y. Svirko, Sci. Rep. 2014, 4, 7191.
[6] K. Batrakov, P. Kuzhir, S. Maksimenko, N. Volynets, S. Voronovich, A. Paddubskaya, G. Valusis, T. Kaplas, Y. Svirko, P. Lambin, Appl. Phys. Lett. 2016, 108, 123101.

[7] M. Lobet, B. Majerus, L. Henrard, P. Lambin, Phys. Rev. B 2016, 93, 235424.

[8] P. P. Kuzhir, A. G. Paddubskaya, N. I. Volynets, K. G. Batrakov, T. Kaplas, P. Lamberti, R. Kotsilkova, P. Lambin, J. Nanophoton. 2017, 11, 032504.

[9] K. S. Novoselov, A. K. Geim, S. V. Morozov, D. Jiang, Y. Zhang, S. V. Dubonos, I. V. Grigorieva, A. A. Firsov, Science 2004, 306, 666.

[10] S. Das Sarma, S. Adam, E. H. Hwang, E. Rossi, Rev. Mod. Phys. 2011, 83, 407.

[11] A. J. Frenzel, C. H. Lui, Y. C. Shin, J. Kong, N. Gedik, Phys. Rev. Lett. 2014, 113, 056602.

[12] L. A. Falkovsky, A. A. Varlamov, Eur. Phys. J. B 2007, 56, 281.

[13] B. Amorim, A. Cortijo, F. de Juan, A. Grushin, F. Guinea, A. Gutierrez-Rubio, H. Ochoa, V. Parente, R. Roldan, P. San-Jose, J. Schiefele, M. Sturla, M. Vozmediano, Phys. Rep. 2016, 617, 1.

[14] V. N. Popov, P. Lambin, Phys. Rev. B 2013, 87, 155425.

[15] S. Y. Li, K. K. Bai, L. J. Yin, J. B. Qiao, W. X. Wang, L. He, Phys. Rev. B 2015, 92, 245302.

[16] C. Si, Z. Sun, F. Liu, Nanoscale 2016, 8, 3207.

[17] V. M. Pereira, R. M. Ribeiro, N. M. R. Peres, A. H. C. Neto, EPL (Europhys. Lett.) 2010, 92, 67001.

[18] M. Bokdam, P. A. Khomyakov, G. Brocks, Z. Zhong, P. J. Kelly, Nano Lett. 2011, 11, 4631.

[19] Y. Zhang, V. W. Brar, C. Girit, A. Zettl, M. F. Crommie, Nat. Phys. $2009,5,722$.

[20] J. Xue, J. Sanchez-Yamagishi, D. Bulmash, P. Jacquod, A. Deshpande, K. Watanabe, T. Taniguchi, P. Jarillo- Herrero, B. J. LeRoy, Nat. Mater. 2011, 10, 282.

[21] R. Decker, Y. Wang, V. W. Brar, W. Regan, H. Z. Tsai, Q. Wu, W. Gannett, A. Zettl, M. F. Crommie, Nano Lett. 2011, 11, 2291.

[22] Y. Zhang, V. W. Brar, F. Wang, C. Girit, Y. Yayon, M. Panlasigui, A. Zettl, M. F. Crommie, Nat. Phys. 2008, 4, 627.

[23] Y. J. Yu, Y. Zhao, S. Ryu, L. E. Brus, K. S. Kim, P. Kim, Nano Lett. 2009, 9,3430 .

[24] T. Cusati, G. Fiori, A. Gahoi, V. Passi, M. C. Lemme, A. Fortunelli, G. Iannaccone, Sci. Rep. 2017, 7, 5109.

[25] H. Liu, H. Kondo, T. Ohno, Phys. Rev. B 2012, 86, 155434.

[26] S. Kim, J. Nah, I. Jo, D. Shahrjerdi, L. Colombo, Z. Yao, E. Tutuc, S. K. Banerjee, Appl. Phys. Lett. 2009, 94, 062107.

[27] T. Chu, Z. Chen, ACS Nano 2014, 8, 3584.

[28] K. Nagashio, T. Nishimura, K. Kita, A. Toriumi, Appl. Phys. Lett. 2010, 97, 143514.

[29] C. Gong, S. McDonnell, X. Qin, A. Azcatl, H. Dong, Y. J. Chabal, K. Cho, R. M. Wallace, ACS Nano 2014, 8, 642.

[30] E. J. H. Lee, K. Balasubramanian, R. T. Weitz, M. Burghard, K. Kern, Nat. Nanotechnol. 2008, 3, 486.

[31] W. S. Leong, H. Gong, J. T. L. Thong, ACS Nano 2014, 8, 994.

[32] T. Ando, J. Phys. Soc. Jpn. 2006, 75, 074716.

[33] E. H. Hwang, S. Das Sarma, Phys. Rev. B 2009, 79, 165404.

[34] P. Hohenberg, W. Kohn, Phys. Rev. 1964, 136, B864.

[35] W. Kohn, L. J. Sham, Phys. Rev. 1965, 140, A1133.

[36] P. Giannozzi, S. Baroni, N. Bonini, M. Calandra, R. Car, C. Cavazzoni, D. Ceresoli, G. L. Chiarotti, M. Cococcioni, I. Dabo, A. Dal Corso, S. de Gironcoli, S. Fabris, G. Fratesi, R. Gebauer, U. Gerstmann, C. Gougoussis, A. Kokalj, M. Lazzeri, L. Martin-Samos, N. Marzari, F. Mauri, R. Mazzarello, S. Paolini, A. Pasquarello, L. Paulatto, C. Sbraccia, S. Scandolo, G. Sclauzero, A. P. Seitsonen, A. Smogunov, P. Umari, R. M. Wentzcovitch, J. Phys.: Condens. Matter 2009, 21, 395502.

[37] J. P. Perdew, K. Burke, M. Ernzerhof, Phys. Rev. Lett. 1996, 77, 3865. 
[38] V. M. Pereira, A. H. Castro Neto, N. M. R. Peres, Phys. Rev. B 2009, $80,045401$.

[39] R. R. Nair, P. Blake, A. N. Grigorenko, K. S. Novoselov, T. J. Booth, T. Stauber, N. M. R. Peres, A. K. Geim, Science 2008, 320, 1308.

[40] F. Bechstedt, L. Matthes, P. Gori, O. Pulci, Appl. Phys. Lett. 2012, 100, 261906.
[41] L. Matthes, P. Gori, O. Pulci, F. Bechstedt, Phys. Rev. B 2013, 87, 035438.

[42] L. Gong, I. A. Kinloch, R. J. Young, I. Riaz, R. Jalil, K. S. Novoselov, Adv. Mater. 2010, 22, 2694.

[43] R. Kotsilkova, P. Todorov, E. Ivanov, T. Kaplas, Y. Svirko, A. Paddubskaya, P. Kuzhir, Carbon 2016, 100, 355. 